Produto \& Produção, vol. 21, n.2, p.01-19. 2020

RECEBIDO EM 16/12/2018. ACEITO EM 24/02/2020.

\author{
Diana Chaukat Chaib \\ Universidade Federal de Alfenas, Programa de Pós-Graduação em Economia, Brasil \\ dianachaib@hotmail.com \\ Jander Luiz Silva Archanjo \\ Universidade Federal de Alfenas, Programa de Pós-Graduação em Economia, Brasil \\ jander.archanjo@gmail.com \\ Vicente Alves Toledo \\ Universidade Federal de Alfenas, Programa de Pós-Graduação em Economia, Brasil \\ vicenteatoledo@gmail.com \\ Marcelo Lacerda Rezende \\ Universidade Federal de Alfenas, Departamento de Ciências Exatas, Brasil \\ marcelo.I.rezende@gmail.com
}

\title{
REVISÃo SISTEMÁTICA DE ESTUDOS SOBRE SUPPLY CHAIN NO BRASIL
}

\section{Resumo}

O conceito Supply Chain ou cadeia de suprimentos exprime a ideia de uma rede de empresas independentes que agem em sintonia de forma a construir valor para o usuário final por meio da distribuição de produtos. Diante da importância do tema, este estudo tem o objetivo de realizar uma revisão sistemática de literatura (RSL), no período entre 2012 e 2018, buscando identificar as características das publicações que discorrem sobre as práticas que envolvem Supply Chain no Brasil. Foram analisados 56 artigos e os resultados evidenciam uma média anual de publicação entre 2012 e 2018 de 8 publicações. "Gerenciamento" constituiu a área temática de $67,8 \%$ das publicações. O periódico que mais publicou foi a "Revista de Administração de Empresas". Sobre as abordagens teóricas realizadas pelos autores, se destacaram "Gestão da Cadeia de Suprimentos" e "Desempenho". Além disso, a cadeia automotiva foi a principal cadeia estudada pelos pesquisadores.

Palavras-Chave: Supply Chain. Cadeia de Suprimentos. Revisão Sistemática de Literatura.

\footnotetext{
Abstract

The concept Supply Chain expresses the idea of a network of independent companies that act in tune to build value for the end user through the distribution of products. Due to the importance of the theme, the study aims to make a systematic literature review, between 2012 and 2018, seeking to identify the characteristics of the publications that discuss the practices that involve Supply Chain in Brazil. It was
} 
analyzed 56 articles and the results show an annual average of publication between 2012 and 2018 of 8 publications. "Management" constituted the thematic area of $67.8 \%$ of publications. The magazine that published the most was the "Revista de Administração de Empresas". Regarding the theoretical approaches carried out by the authors, the following stand out: "Supply Chain Management" and "Performance". In addition, the automotive chain was the main chain studied by the researchers.

\section{Introdução.}

O período que compreende as décadas de 1980 e 2000 foi marcado por grandes transformações nos conceitos gerenciais, especialmente no que se refere à função de operações (FLEURRY, 1999). Um desses conceitos é o Supply Chain, ou cadeia de suprimentos. A cadeia de suprimentos deve ser vista como uma rede de empresas independentes que agem em sintonia de forma a construir valor para o usuário final por meio da distribuição de produtos e, em particular, essa sintonia é a finalidade da gestão de cadeia de suprimentos (BATALHA, 1999).

Diante dessa questão, torna-se perceptível a importância do tema da cadeia de suprimentos e que seu campo de estudo está em fase de desenvolvimento, não só em âmbito acadêmico, mas também organizacional. Assim, estudos sobre este conceito são oportunos e necessários para ajudar a melhorar a compreensão de suas pressões, a aplicação de suas práticas e o conhecimento de seus resultados alcançados (ZHU; SARKIS; LAI, 2008).

Sendo assim, esse estudo tem como principal objetivo realizar uma revisão sistemática da literatura (RSL) existente sobre Supply Chain a fim de verificar as características da produção científica sobre cadeias de suprimento no Brasil.

Esta pesquisa está estruturada em 5 seções, incluindo esta introdução. A segunda seção apresenta uma breve conceituação do termo pesquisado Supply Chain. A próima seção apresenta os procedimentos metodológicos utilizados na pesquisa, em que se explica como a revisão sistemática da literatura foi realizada. Em seguida, tem-se a apresentação e a análise dos resultados das buscas na base de dado utilizada. Na última seção, são feitas algumas considerações finais.

\section{Supply Chain.}

Uma cadeia de suprimento é responsável por agregar diversos agentes econômicos e institucionais, desde os fornecedores de matérias-primas, indústrias de transformação, distribuidores, varejistas até os consumidores finais (SCRAMIM; BATALHA 1999). Nesse sentido, Supply Chain, ou cadeia de suprimentos deve ser vista como uma rede de empresas independentes que agem em sintonia de forma a construir valor para o usuário final por meio da distribuição de produtos e, em particular, essa sintonia é a finalidade da gestão de cadeia de suprimentos (BATALHA, 1999).

É dentro deste contexto que surge o conceito de Gestão da Cadeia de Suprimentos (GCS). O Supply Chain Management, ou GCS, começou a se desenvolver apenas no início dos anos 90 (FLEURRY, 1999) e busca promover entre os agentes de uma cadeia produtiva ou rede de empresas uma relação de benefícios mútuos pela definição de estruturas organizacionais e relações contratuais (SCRAMIM; BATALHA 1999).

Uma questão importante, apresentada na literatura existente sobre a gestão de cadeias de suprimento, gira em torno da dificuldade de identificar esses ganhos e benefícios mútuos aos agentes econômicos pela aplicação desse conceito (SCRAMIM; BATALHA, 2010). 
Gilbert e Ballou (1999) defendem que a melhoria da coordenação, em uma cadeia de suprimento, envolve a tentativa de um agente de mudar o comportamento de pelo menos um agente, por meio de algum tipo de incentivo. Na mesma direção, Zimmer (2002) destaca que embora o custo global de uma cadeia de suprimento seja minimizado, pode ocorrer uma situação na qual o custo de um agente seja reduzido, enquanto o custo de outro aumente. Esses autores destacam a questão da melhoria da performance operacional, por meio da redução de custos dos agentes, e do sistema como um todo, como crucial para ganho de competitividade.

Apesar do crescente interesse, nota-se uma certa diversidade de classificações e enquadramentos de trabalhos e pesquisas, seja pela mídia, pela academia e também pelo mundo corporativo em que os temas tratados entre cadeias de suprimentos aparecem interligados com questões ambientais e sociais (BRITO; BERARDI, 2010).

\section{Metodologia.}

A metodologia que orientou os procedimentos técnicos seguidos neste estudo, tal como o método de investigação, possibilita a sua classificação como uma pesquisa explicativa, baseada no método de revisão de literatura (MARINI et al., 2012). A revisão sistemática de literatura (RSL) é um método de pesquisa para busca e análise de artigos de determina área da ciência. Esse método busca responder questões teóricas por meio da análise de todo o conhecimento acumulado por pesquisadores no conjunto de artigos existentes sobre essa determinada área. (CONFORTO; AMARAL; SILVA, 2011)

Mulrow (1994) enfatiza que revisões sistemáticas são atividades pouco valorizadas no mundo científico. No entanto, setores como saúde, estudos demográficos e legisladores de políticas públicas necessitam fazer uso desse método para integrar e analisar de forma eficiente uma grande quantidade de informação, possibilitando uma tomada de decisão com maior eficácia. Para Mulrow (1994), o uso de procedimentos sistemáticos aumenta a confiabilidade e acuracidade das conclusões e resultados do estudo. Completa argumentando que, apesar da revisão sistemática consumir bastante tempo e recursos, ainda assim é mais rápido e demanda menos custos do que iniciar um novo estudo em uma área que já possui resultados publicados, mas que não foram devidamente explorados (MULROW, 1994).

Para que a revisão sistemática de literatura seja feita com rigor metodológico, é imprescindível levar em consideração alguns fatores. Em um primeiro momento, é necessário definir o problema e os objetivos da pesquisa, selecionar as fontes primárias para ter o embasamento teórico sobre o tema que está sendo pesquisado, estabelecer critérios para a seleção dos artigos pertinentes e, por último, obter a síntese dos resultados obtidos com a adoção da RSL. (CONFORTO; AMARAL; SILVA, 2011)

O objetivo desta RSL é identificar os artigos publicados que discorrem sobre as práticas que envolvem Supply Chain no Brasil. Para tanto, foram usados alguns critérios na busca de pesquisas nessa área.

\subsection{Strings de busca.}

As buscas da revisão sistemática de literatura foram realizadas na base de dados SCIELO, traçando como horizonte de tempo Janeiro de 2012 até Junho de 2018. O quadro 1 mostra a base de dados utilizada, a data da pesquisa, as palavras-chave, os campos considerados nas buscas (título, resumo, palavras-chave). 
Os critérios de inclusão foram denominados Filtros 1 e 2 e também estão indicados no quadro 1. O Filtro 1 foi realizado na própria base de dados, atentando para aspectos como área temática e ano de publicação. O Filtro 2 consistiu na leitura dos títulos e resumos dos artigos para identificar se o estudo considerava realmente as relações buscadas (supply chain).

\subsection{Critérios de qualificação.}

Nesse estudo não foram usados critérios de qualificação, uma vez que a quantidade de artigos resultantes das buscas foi suficiente para viabilizar a leitura e análise dos documentos. Sendo assim, todos os artigos encontrados na pesquisa foram submetidos ao Filtro 1 e, em seguida, ao Filtro 2, conforme os critérios de inclusão comentados acima.

\subsection{Método e ferramentas.}

Nas buscas realizadas foram utilizados os mesmos métodos e ferramentas descritos a seguir. Pelo Filtro 1, foram considerados para análise os trabalhos da área temática de "Ciências Sociais Aplicadas" publicadas de janeiro de 2012 até junho de 2018. A área temática "Ciências Sociais Aplicadas" foi utilizado como critério de inclusão com o objetivo de se alcançar os trabalhos que analisam a cadeia de suprimento levando em consideração, em alguma medida, o contexto social de onde está inserida a cadeia.

O Filtro 2 consistiu na leitura dos títulos e resumos dos artigos resultantes do Filtro 1, tendo como objetivo identificar se o artigo tratava do tema supply chain no contexto brasileiro. A partir disso fez-se o download dos artigos resultantes do Filtro 2. Na primeira busca, após aplicação do Filtro 1, foram encontrados um total de 142 resultados. Após a aplicação dos filtros, encaixaram-se 56pesquisas para análise (ANEXO A).

\subsection{Forma de classificação dos artigos.}

Os 56 artigos selecionados após a aplicação dos filtros definidos acima foram classificados de acordo com o ano de publicação, o periódico publicado, a área de conhecimento, o método de pesquisa, a abordagem teórica, o objeto da pesquisa e o tipo de cadeia analisada. A classificação de acordo com estes componentes foi feita por meio das leituras de título, resumo e palavras-chave, e quando necessário, buscou-se ao longo do texto algum componente não encontrado com a primeira leitura.

Após a aplicação dos filtros, as publicações analisadas se deram em 19 periódicos, quais sejam: Revista de Administração de Empresas, Journal of Techonology Management \& Innovation, Revista de Administração Contemporânea, Brazilian Administration Review, Revista Eletrônica de Administração, Revista de Administração, Revista de Administração Mackenzie, Interações, Revista de Gestão da Tecnologia e Sistemas de Informação, Revista de Administração Pública, Economia e Sociedade, Mundo Agrário, Cuadernos de Desarollo Rural, Revista Brasileira de Gestão e Negócios, Nova Economia, Brazilian Journal of Political Economy, Organizações e Sociedade, Revista de Economia e Sociologia Rural e, por fim, Brazilian Business Review.

A área de conhecimento foi classificada de acordo com a área temática das publicações, sendo composta por: Gerenciamento, Engenharia, Planejamento e Desenvolvimento, Economia, Agricultura, Administração Pública e, por último Economia e Política Agrícola. Vale ressaltar que a área temática foi identificada na própria base de dados utilizada, Scielo. 
Os métodos de pesquisa identificados, por sua vez, foram: Survey, Estudo de Caso, Estudo de Caso/Survey, Conceitual/Teórico/Revisão Sistemática de Literatura e Modelagem.

A abordagem teórica foi classificada em 12 temas, sendo: Gestão da Cadeia de Suprimentos, Desempenho, Tecnologia de Informação, Sustentabilidade, Gestão Ambiental, Estratégia, Colaboração, Logística, Desenvolvimento, Preferências do Consumidor, Práticas Organizacionais, e Instituições.

O objeto da pesquisa é o assunto principal de cada artigo e foi utilizado para classificar a abordagem teórica. Por fim, o tipo de cadeia foi especificado para os artigos aplicados a determinada cadeia de suprimentos.

Quadro 1 - Strings de busca

\begin{tabular}{|c|c|c|c|c|c|c|}
\hline $\begin{array}{l}\text { Base de } \\
\text { dados }\end{array}$ & $\begin{array}{l}\text { Palavras- } \\
\text { Chave }\end{array}$ & $\begin{array}{l}\text { Resultado } \\
\text { parcial }\end{array}$ & Filtro 1 & $\begin{array}{l}\text { Resultado } \\
\text { parcial } 2\end{array}$ & Filtro 2 & $\begin{array}{l}\text { Resultado } \\
\text { final }\end{array}$ \\
\hline SCIELO & $\begin{array}{l}\text { "Cadeia de } \\
\text { Suprimentos" } \\
\text { ou "Supply } \\
\text { Chain" }\end{array}$ & 850 & $\begin{array}{l}\text { Ciências } \\
\text { Sociais } \\
\text { Aplicadas } \\
\text { Ano de } \\
\text { publicação: } \\
2012 \text { a } 2018\end{array}$ & 142 & $\begin{array}{l}\text { Título, } \\
\text { resumo e } \\
\text { palavras- } \\
\text { chave }\end{array}$ & 56 \\
\hline
\end{tabular}

Fonte: Dados da pesquisa. Elaboração própria 


\section{Resultados.}

\subsection{Evolução das Publicações no Período.}

A evolução das publicações sobre Cadeia de Suprimentos no Brasil ao longo do período analisado pode ser observada no Gráfico 1. Foi verificada uma média de 8 publicações por ano, com destaque para 2014 e 2015 que concentram o maior número, com 16 e 19 artigos publicados, respectivamente. Estes dois anos representam quase metade do total de artigos publicados sobre o tema em estudo de 2012 a 2017. Vale dizer que a média foi feita para o período analisado no gráfico com a ressalva do ano de 2018, uma vez que os estudos deste ano compreendem somente o primeiro semestre.

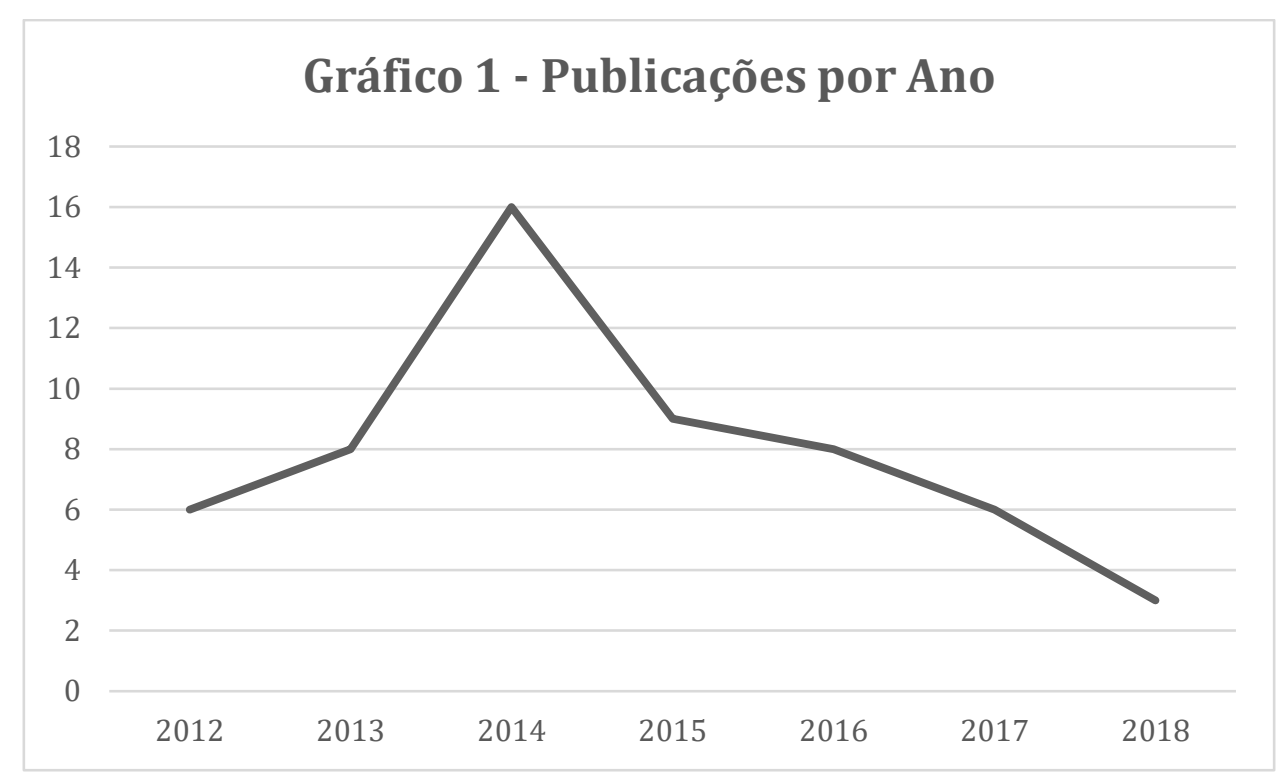

Fonte: Dados da Pesquisa. Elaboração Própria.

\subsection{Periódicos.}

No que diz respeito à publicação por periódico, como pode ser observado no Gráfico 2 , os periódicos com maior número de publicações foram a Revista de Administração de Empresas com 8 publicações, seguida por Journal of Technology Management \& Innovation com 7 publicações e Revista de Administração Contemporânea com 6 publicações. Estas revistas representaram 37,5\% dos artigos publicados sobre o tema em questão entre 2012 a 2018. 


\section{Gráfico 2 - Publicações Por Periódico}

Revista de Administração de Empresas Journal of technology management \& innovation

Revista de Administração Contemporânea

Brazilian Administration Review

Revista Eletrônica de Administração

Revista de Administração

Revista de Administração Mackenzie Interações

Revista de Gestão da Tecnologia e Sistemas de...

Revista de Administração Pública

Economia e Sociedade Mundo Agrario

Cuadernos de Desarrollo Rural Revista Brasileira de Gestão e Negócios Nova Economia

Brazilian Journal of Political Economy

Organizações e Sociedade

Revista de Economia e Sociologia Rural

Brazilian Business Review

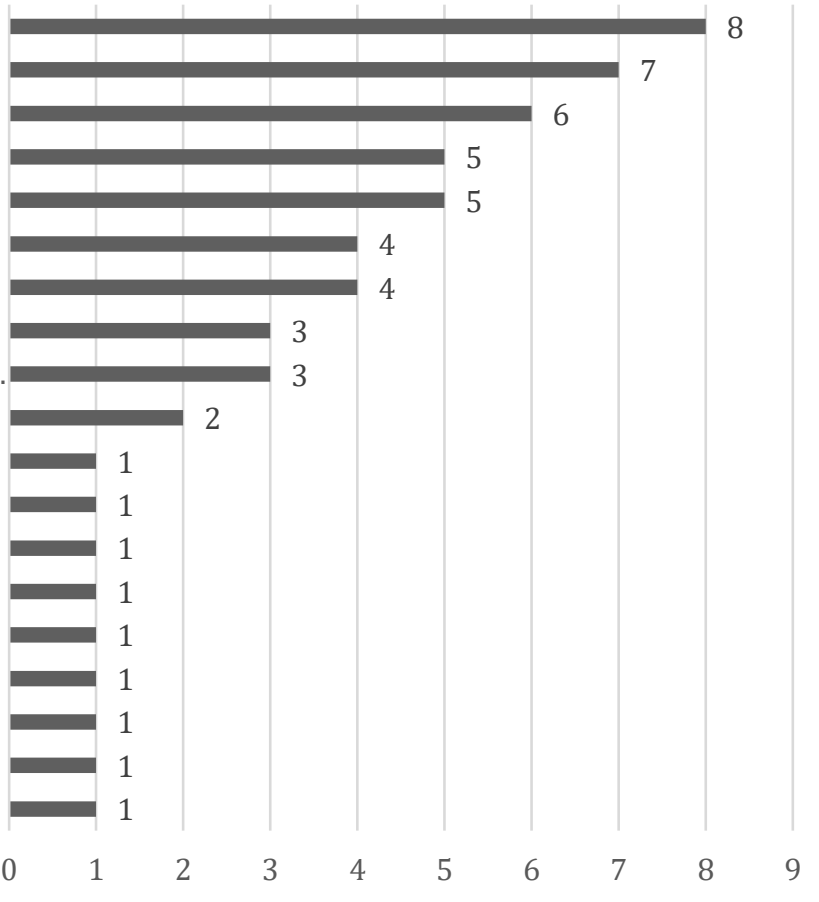

Fonte: Dados da Pesquisa. Elaboração Própria.

Ocupando uma posição intermediária quanto ao número de artigos publicados estão as revistas Brazilian Administration Review, Revista Eletrônica de Administração, com 5 publicações cada, e, Revista de Administração e Revista de Administração Mackenzie com 4. Estes cinco periódicos representam $32,1 \%$ do total de artigos das revistas.

Os 11 periódicos restantes publicaram menos de 4 artigos sobre Cadeia de Suprimentos ao longo do período considerado, 9 destes contendo apenas 1 artigo. 


\section{3. Área de Conhecimento.}

Quanto a área temática, observa-se pelo Gráfico 3 que "Gerenciamento" constitui a área temática de 67,8\% das publicações analisadas, seguido por "Engenharia", área temática de 7 trabalhos analisados.

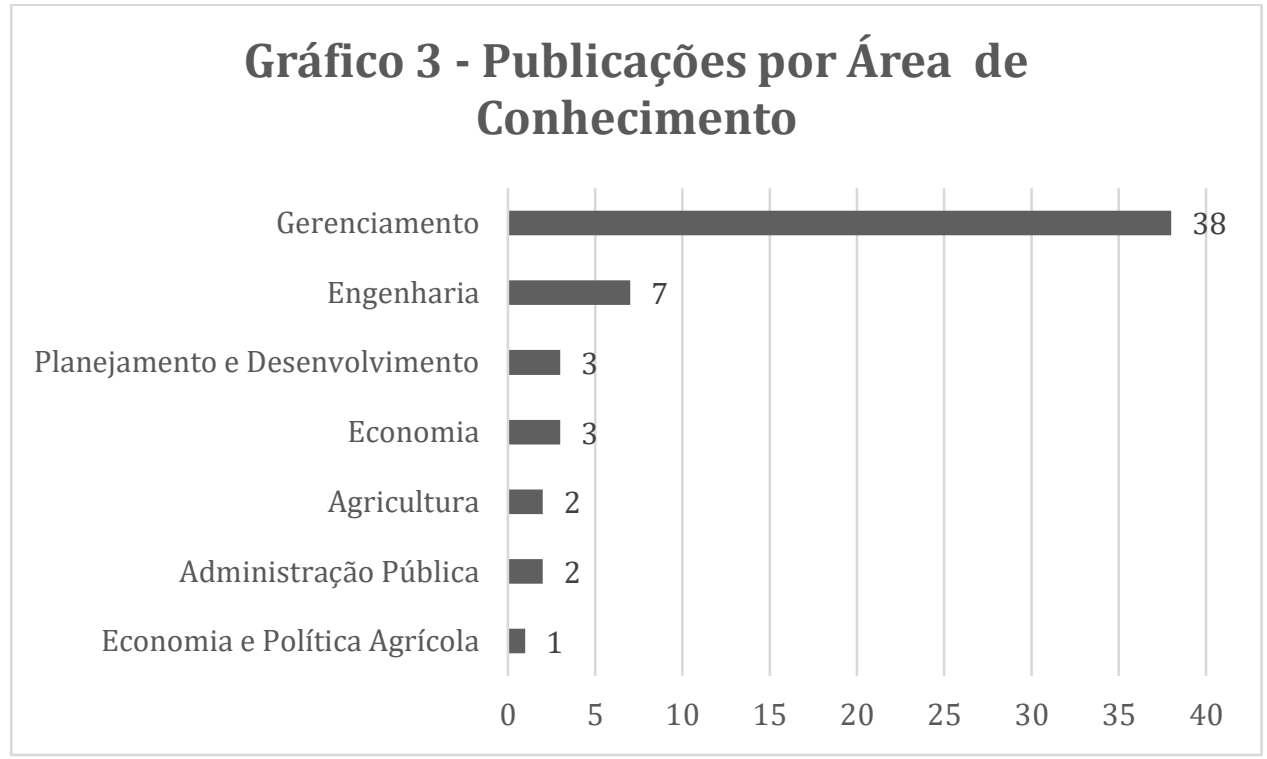

Fonte: Dados da Pesquisa. Elaboração Própria.

\subsection{Método de pesquisa.}

Os resultados indicam que a metodologia mais utilizada pelos trabalhos analisados foi Survey, seguido por estudo de caso.

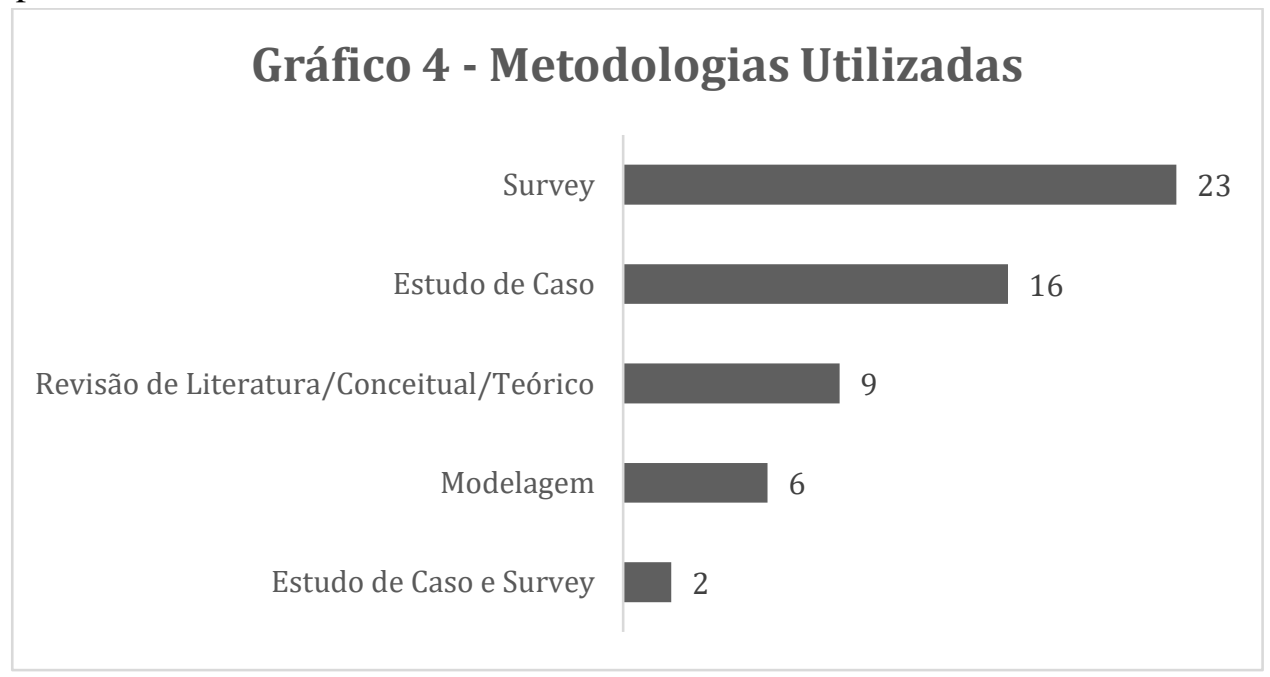

Fonte: Dados da Pesquisa. Elaboração Própria.

Esse resultado evidencia que a grande parte das pesquisas publicadas sobre Cadeia de Suprimentos no Brasil durante o período em análise desenvolveram estudos empíricos. Isto indica que a maioria dos trabalhos sobre o tema investigado procurou aplicar conhecimentos e teorias. 


\subsection{Abordagem Teórica.}

A classificação dos artigos quanto à abordagem teórica contribui para situar o objeto de pesquisa dos mesmos em um determinado tema. As diversas abordagens teóricas realizadas pelos autores, podem ser verificadas no Gráfico 4. De acordo com a pesquisa realizada, a "Gestão da Cadeia de Suprimentos" e "Desempenho" foram as principais abordagens realizadas, sendo cada uma abordada 9 vezes, constituindo juntas $32,1 \%$ do total de trabalhos selecionados na pesquisa, seguidas por "Tecnologia de Informação" e "Sustentabilidade", abordadas 6 vezes cada.

Os artigos pertencentes à abordagem teórica mais recorrente "Gestão da Cadeia de Suprimentos" foram publicados de 2013 a 2016, enquanto os artigos da outra abordagem mais utilizada foram publicados em todos os anos completos considerados na análise (2012, 2013, 2014, 2015, 2016 e 2017). Isto ocorre de maneira similar para as segundas abordagens mais realizadas, em que os artigos sobre "Tecnologia da Informação" foram publicados no mesmo intervalo de quatro anos apontado acima, enquanto as pesquisas sobre "Sustentabilidade" foram publicadas em quase todos anos.

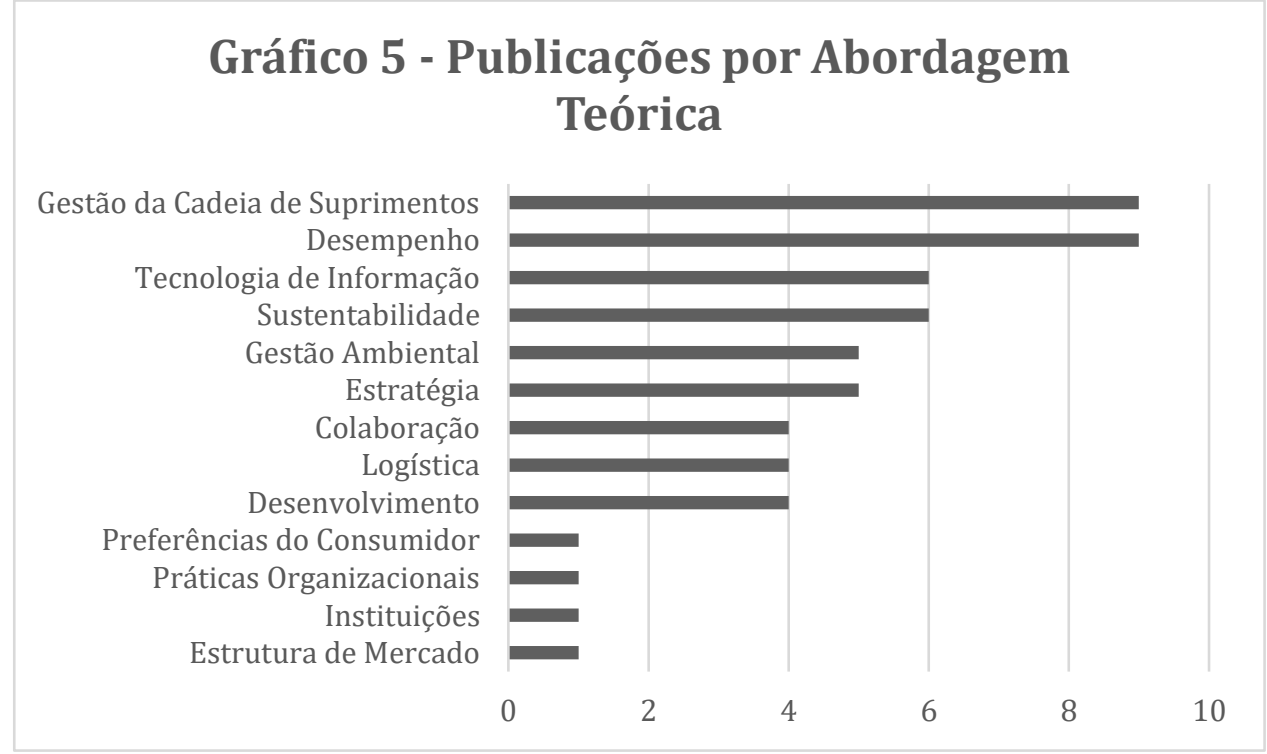

Fonte: Dados da Pesquisa. Elaboração Própria. 
4.5.1. Abordagens Específicas.

No quadro a seguir, são relacionados os objetos de pesquisa dos trabalhos analisados e suas correspondentes abordagens teóricas.

Quadro 2 - Abordagens Teóricas e Objeto de Pesquisa dos Trabalhos

\begin{tabular}{|c|c|c|c|c|}
\hline Abordagem Teórica & \multicolumn{4}{|c|}{ Abordagem Específica } \\
\hline 1. Colaboração & $\begin{array}{c}\text { Relações de } \\
\text { confiança entre } \\
\text { os fornecedores }\end{array}$ & $\begin{array}{l}\text { Relação entre } \\
\text { cooperação e } \\
\text { desempenho }\end{array}$ & $\begin{array}{c}\text { Vantagens } \\
\text { competitivas } \\
\text { proveniente da } \\
\text { cooperação }\end{array}$ & $\begin{array}{l}\text { Identificação de } \\
\text { métodos } \\
\text { colaborativos }\end{array}$ \\
\hline \multirow[t]{2}{*}{ 2. Desempenho } & $\begin{array}{c}\text { Efeitos da } \\
\text { dependência } \\
\text { organizacional } \\
\text { sobre as } \\
\text { capacidades da } \\
\text { firma }\end{array}$ & $\begin{array}{c}\text { Efeitos da } \\
\text { imersão } \\
\text { relacional e da } \\
\text { imersão } \\
\text { estrutural no } \\
\text { desempenho } \\
\text { operacional das } \\
\text { firmas }\end{array}$ & $\begin{array}{c}\text { Efeitos da } \\
\text { tecnologia no } \\
\text { desempenho }\end{array}$ & $\begin{array}{c}\text { Capacidade } \\
\text { tecnológica dos } \\
\text { ofertantes da } \\
\text { indústria de } \\
\text { construção } \\
\text { naval }\end{array}$ \\
\hline & $\begin{array}{c}\text { Impacto do } \\
\text { fenômeno } \\
\text { "hockey stick" } \\
\text { no desempenho } \\
\text { financeiro }\end{array}$ & $\begin{array}{c}\text { Relação entre } \\
\text { flexibilidade e } \\
\text { capacidade da } \\
\text { empresa atender } \\
\text { demanda }\end{array}$ & $\begin{array}{c}\text { Correlação } \\
\text { entre o } \\
\text { desempenho } \\
\text { produtivo e } \\
\text { capabilidade }\end{array}$ & \\
\hline 3. Desenvolvimento & $\begin{array}{c}\text { Política para o } \\
\text { desenvolviment } \\
\text { o da cadeia }\end{array}$ & $\begin{array}{c}\text { Capacidade das } \\
\text { exportações de } \\
\text { commodities em } \\
\text { promover } \\
\text { desenvolvimento }\end{array}$ & $\begin{array}{c}\text { Papel da cadeia } \\
\text { no } \\
\text { desenvolviment } \\
\text { o regional }\end{array}$ & $\begin{array}{c}\text { Papel do } \\
\text { sistema } \\
\text { agroalimentar } \\
\text { no } \\
\text { desenvolvimen } \\
\text { to rural }\end{array}$ \\
\hline \multirow[t]{2}{*}{ 4. Estratégia } & $\begin{array}{l}\text { Estratégias } \\
\text { frente à } \\
\text { restrição de } \\
\text { oferta }\end{array}$ & $\begin{array}{c}\text { Conflitos } \\
\text { existentes na } \\
\text { cadeia e } \\
\text { comportamento } \\
\text { estratégico pelas } \\
\text { empresas }\end{array}$ & $\begin{array}{l}\text { Vantagem } \\
\text { competitiva } \\
\text { sustentável }\end{array}$ & \\
\hline & $\begin{array}{l}\text { Alinhamento da } \\
\text { estratégia } \\
\text { organizacional }\end{array}$ & $\begin{array}{c}\text { Estratégia dos } \\
\text { principais }\end{array}$ & & \\
\hline
\end{tabular}




\begin{tabular}{|c|c|c|c|c|}
\hline & $\begin{array}{c}\text { pelos elos da } \\
\text { cadeia }\end{array}$ & $\begin{array}{l}\text { agentes da } \\
\text { cadeia }\end{array}$ & & \\
\hline $\begin{array}{l}\text { 5. Estrutura de } \\
\text { Mercado }\end{array}$ & $\begin{array}{l}\text { Identificação de } \\
\text { poder de } \\
\text { mercado }\end{array}$ & & & \\
\hline $\begin{array}{l}\text { 6. Gestão } \\
\text { Ambiental }\end{array}$ & $\begin{array}{l}\text { Práticas de } \\
\text { gestão } \\
\text { ambiental }\end{array}$ & $\begin{array}{c}\text { Barreiras à } \\
\text { gestão ambiental } \\
\text { da cadeia }\end{array}$ & & \\
\hline \multirow{2}{*}{$\begin{array}{l}\text { 7. Gestão da Cadeia } \\
\text { de Suprimentos }\end{array}$} & $\begin{array}{l}\text { Relação entre a } \\
\text { coordenação } \\
\text { dos negócios e o } \\
\text { suprimento do } \\
\text { produto }\end{array}$ & $\begin{array}{l}\text { Interação entre } \\
\text { as empresas }\end{array}$ & $\begin{array}{c}\text { Identificação } \\
\text { dos atributos } \\
\text { que precisam } \\
\text { ser melhorados } \\
\text { ao longo da } \\
\text { cadeia }\end{array}$ & $\begin{array}{c}\text { Gestão de } \\
\text { custos }\end{array}$ \\
\hline & $\begin{array}{l}\text { Avaliação e a } \\
\text { aplicabilidade } \\
\text { de um modelo } \\
\text { de Gestão da } \\
\text { Cadeia de } \\
\text { Suprimentos } \\
\text { (GCS) }\end{array}$ & $\begin{array}{c}\text { Estrutura e } \\
\text { coordenação da } \\
\text { cadeia }\end{array}$ & $\begin{array}{l}\text { Critérios e } \\
\text { métodos para } \\
\text { seleção de } \\
\text { fornecedores }\end{array}$ & \\
\hline 8. Instituições & $\begin{array}{c}\text { Papel do } \\
\text { judiciário nas } \\
\text { disputas }\end{array}$ & & & \\
\hline 9. Logística & $\begin{array}{l}\text { Estratégia de } \\
\text { logística }\end{array}$ & $\begin{array}{c}\text { Efeito do tipo do } \\
\text { produto } \\
\text { exportado e das } \\
\text { capacidades } \\
\text { logísticas sobre o } \\
\text { ciclo de entrega } \\
\text { de pedido }\end{array}$ & $\begin{array}{c}\text { Efeito das } \\
\text { capacidades } \\
\text { logísticas sobre } \\
\text { a resiliência da } \\
\text { cadeia de } \\
\text { suprimentos }\end{array}$ & \\
\hline $\begin{array}{l}\text { 10. Práticas } \\
\text { Organizacionais }\end{array}$ & $\begin{array}{l}\text { Práticas dos } \\
\text { funcionários } \\
\text { dos níveis } \\
\text { estratégico, } \\
\text { tático e } \\
\text { operacional }\end{array}$ & & & \\
\hline $\begin{array}{l}\text { 11. Preferências do } \\
\text { Consumidor }\end{array}$ & $\begin{array}{c}\text { Percepção das } \\
\text { preferências dos } \\
\text { consumidores } \\
\text { por parte dos }\end{array}$ & & & \\
\hline
\end{tabular}




\begin{tabular}{|c|c|c|c|c|}
\hline & $\begin{array}{l}\text { agentes da } \\
\text { cadeia }\end{array}$ & & & \\
\hline \multirow[t]{2}{*}{ 12. Sustentabilidade } & $\begin{array}{c}\text { Papel da } \\
\text { mobilização } \\
\text { social na } \\
\text { reestruturação } \\
\text { produtiva } \\
\text { sustentável }\end{array}$ & $\begin{array}{c}\text { Papel da } \\
\text { colaboração e } \\
\text { dos incentivos na } \\
\text { sustentabilidade }\end{array}$ & $\begin{array}{c}\text { Estratégias } \\
\text { para } \\
\text { sustentabilidad } \\
\text { e }\end{array}$ & \\
\hline & $\begin{array}{c}\text { Efeito da } \\
\text { inovação sobre } \\
\text { a } \\
\text { sustentabilidade } \\
\text { da cadeia }\end{array}$ & $\begin{array}{c}\text { Determinantes } \\
\text { da } \\
\text { sustentabilidade } \\
\text { e } \\
\text { competitividade } \\
\text { da cadeia }\end{array}$ & $\begin{array}{c}\text { Papel da } \\
\text { tecnologia na } \\
\text { sustentabilidad } \\
\text { e }\end{array}$ & \\
\hline $\begin{array}{l}\text { 13. Tecnologia de } \\
\text { Informação }\end{array}$ & $\begin{array}{c}\text { Papel da } \\
\text { tecnologia da } \\
\text { informação na } \\
\text { gestão da cadeia }\end{array}$ & $\begin{array}{c}\text { Uso da } \\
\text { tecnologia da } \\
\text { informação para } \\
\text { melhorar a } \\
\text { colaboração }\end{array}$ & $\begin{array}{c}\text { Práticas e- } \\
\text { procurement }\end{array}$ & $\begin{array}{c}\text { Relação entre } \\
\text { tecnologia de } \\
\text { informação e a } \\
\text { integração na } \\
\text { cadeia de } \\
\text { suprimentos }\end{array}$ \\
\hline
\end{tabular}

Fonte: Dados da pesquisa. Elaboração própria. 
4.6. Tipo de Cadeia de Suprimentos.

O Gráfico 6 evidencia os diversos tipos de cadeia abordados pelas pesquisas. Entre elas, destacam-se como mais abordadas a cadeia automotiva, 5 vezes abordadas e a de carne, 4 vezes.

\section{Gráfico 6 - Tipos de Cadeia de Suprimentos Abordados}

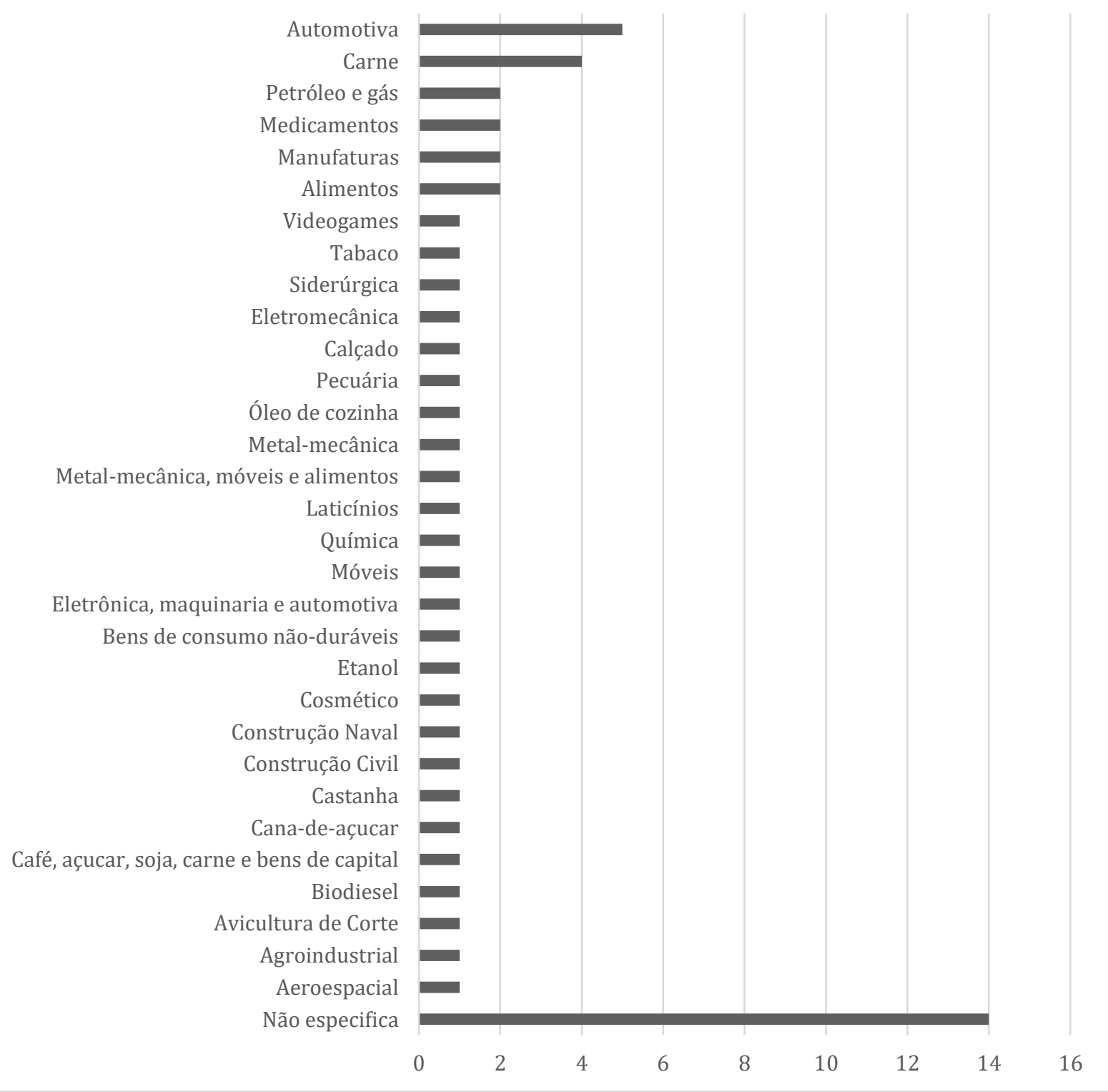

Fonte: Dados da pesquisa. Elaboração Própria. 


\section{Considerações Finais.}

Esse estudo teve como principal objetivo verificar as características da produção científica sobre cadeias de suprimento no Brasil, adotando a metodologia de revisão sistemática da literatura (RSL). Em particular, o objetivo desta RSL foi identificar e analisar os artigos publicados que discorrem sobre as práticas que envolvem Supply Chain. Para tanto, foram usados alguns critérios na busca de pesquisas nessa área.

Os resultados evidenciam que a área temática "Gerenciamento" consiste em 67,8\% das publicações consideradas no estudo. Esse resultado está de acordo com a visão de que o gerenciamento das estruturas organizacionais e relações contratuais das cadeias produtivas pode promover uma relação de benefícios mútuos entre seus agentes, sendo um dos principais objetivos de se estudar a cadeia como um todo. Está também relacionado com as principais abordagens teóricas utilizadas pelos autores, que são a gestão e o desempenho da cadeia de suprimentos.

Os trabalhos foram desenvolvidos em diversos setores (Gráfico 6) demonstrando o potencial desse tipo de estudo como ferramenta de análise. No horizonte de tempo do trabalho, os periódicos que mais publicaram foram Revista de Administração de Empresas, Journal of Technology Management \& Innovation, e Revista de Administração Contemporânea. Sobre a metodologia adotada por esses estudos, é interessante ressaltar que a maioria segue métodos empíricos.

Apesar do crescente interesse nessa área, nota-se uma certa diversidade de classificações e enquadramentos de trabalhos e pesquisas, não só pela academia como também pelo mundo corporativo em que os temas tratados entre cadeias de suprimentos aparecem interligados com questões ambientais e sociais como já observado por Brito e Berardi (2010). Diante dessa questão, torna-se perceptível a importância do tema da cadeia de suprimentos. Além disso, como ressaltado por ZHU; SARKIS; LAI, (2008) seu campo de estudo está em fase de desenvolvimento, não só em âmbito acadêmico, mas também organizacional. Assim, o acompanhamento e análise do conhecimento acumulado pelos pesquisadores no conjunto de artigos existentes pode auxiliar na compreensão desse desenvolvimento e apontar lacunas existentes que precisam ser trabalhadas.

\section{Referências Bibliográficas}

BATALHA, M.O. \& DA SILVA, A.L “Gestão de Cadeias Produtivas: Novos Aportes Teóricos e Empíricos”. mimeo, documento preliminar para discussão. São Carlos, 1999.

BRITO, Renata Peregrino de; BERARDI, Patricia Calicchio. Vantagem competitiva na gestão sustentável da cadeia de suprimentos: um metaestudo. 2010.

CONFORTO, E. C.; AMARAL, D. C.; SILVA, S. L. D. Roteiro para revisão bibliográfica sistemática: aplicação no desenvolvimento de produtos e gerenciamento de projetos. VIII Congresso Brasileiro de Gestão de Desenvolvimento de Produto (CBGDP). Anais... p.1-12, Porto Alegre, 2011.

FLEURY, Paulo Fernando. Supply Chain Management: conceitos, oportunidades e desafios da implementação. Revista Tecnologística, v. 4, n. 30, p. 25-32, 1999.

GILBERT, S. M.; BALLOU, R. H. Supply chain benefits from advanced customer commitments. Journal of Operations Management, v.18, p. 61-73, 1999. 
MARINI, Marcos Junior et al. Avaliação da contribuição de arranjos produtivos locais para o desenvolvimento local. Biblio 3w: revista bibliográfica de geografía y ciencias sociales, v. 17, 2012.

MULROW, C.D. Systematic reviews rationale for systematic reviews. British Medical Journal, v.309, pp.597-599, 1994.

SCRAMIM, Fernando Cezar Leandro; BATALHA, Mário Otávio. Supply Chain Management em cadeias agroindustriais: discussões a cerca das aplicações no setor lácteo brasileiro. Ribeirão Preto: PENSA/FEA/USP, 1999.

SCRAMIM, Fernando Cezar Leandro; BATALHA, Mário Otávio. Método para análise de benefícios em cadeias de suprimento: um estudo de caso. 2010.

ZIMMER, K. Supply chain coordination with uncertain just-in-time delivery. International Journal of Production Economics, v. 77, n. 1, p. 1-15, 2002.

ZHU, Q.; SARKIS, J.; LAI, K.-H. Green supply chain management implications for "closing the loop". Transportation Research Part E: Logistics and Transportation Review, v.44, n.1, p.1-18, jan. 2008.

ANEXO A - Trabalhos Analisados na Pesquisa

\begin{tabular}{|c|c|c|c|}
\hline NOME DO ARTIGO & $\begin{array}{c}\text { ANO DE } \\
\text { PUBLICAÇÃO }\end{array}$ & PERIÓDICO & $\begin{array}{c}\text { ÁREA DE } \\
\text { CONHECIMENTO }\end{array}$ \\
\hline $\begin{array}{c}\text { GESTÃO DA CADEIA DE SUPRIMENTOS VERDE: UMA } \\
\text { ANÁLISE DA RELAÇÃO }\end{array}$ & 2016 & $\begin{array}{c}\text { BRAZILIAN } \\
\text { BUSINESS REVIEW } \\
\text { FORNECEDOR SUL DO BRASIL }\end{array}$ & GERENCIAMENTO \\
\hline $\begin{array}{c}\text { UNDERSTANDING SUPPLY NETWORKS FROM COMPLEX } \\
\text { ADAPTIVE SYSTEMS }\end{array}$ & 2014 & $\begin{array}{c}\text { BRAZILIAN } \\
\text { ADMINISTRATION } \\
\text { REVIEW }\end{array}$ & GERENCIAMENTO \\
\hline $\begin{array}{c}\text { TRUST AND SUPPLIER-BUYER RELATIONSHIPS: AN } \\
\text { EMPIRICAL ANALYSIS }\end{array}$ & 2013 & $\begin{array}{c}\text { BRAZILIAN } \\
\text { ADMINISTRATION } \\
\text { REVIEW }\end{array}$ & GERENCIAMENTO \\
\hline $\begin{array}{c}\text { VALUE ADDED ELEMENTS ACCORDING TO BUYER } \\
\text { COMPANIES IN A B2B CONTEXT }\end{array}$ & 2015 & $\begin{array}{c}\text { BRAZILIAN } \\
\text { ADMINISTRATION } \\
\text { REVIEW }\end{array}$ & GERENCIAMENTO \\
\hline $\begin{array}{c}\text { EFFECTS OF PATH DEPENDENCE ON CAPABILITIES IN } \\
\text { CAPTIVE GLOBAL VALUE CHAINS }\end{array}$ & 2015 & $\begin{array}{c}\text { BRAZILIAN } \\
\text { ADMINISTRATION } \\
\text { REVIEW }\end{array}$ & GERENCIAMENTO \\
\hline $\begin{array}{c}\text { HOCKEY STICK PHENOMENON: SUPPLY CHAIN } \\
\text { MANAGEMENT CHALLENGE IN BRAZIL }\end{array}$ & 2014 & $\begin{array}{c}\text { BRAZILIAN } \\
\text { ADMINISTRATION } \\
\text { REVIEW }\end{array}$ & GERENCIAMENTO \\
\hline $\begin{array}{c}\text { POLÍTICAS DE APOIO À INDÚSTRIA DE PETRÓLLO E GÁS } \\
\text { NO BRASIL: UM ESTUDO DAS AÇÕES PÚBLICAS PARA O } \\
\text { DESENVOLVIMENTO DA CADEIA DE VALOR }\end{array}$ & 2017 & $\begin{array}{c}\text { ECONOMIA E } \\
\text { SOCIEDADE }\end{array}$ & ECONOMIA \\
\hline $\begin{array}{c}\text { CADEIAS PRODUTIVAS SUSTENTÁVEIS NO } \\
\text { DESENVOLVIMENTO TERRITORIAL: A CASTANHA NA } \\
\text { BOLÍVIA E NO ACRE, BRASIL }\end{array}$ & 2016 & $\begin{array}{c}\text { INTERAÇÕES } \\
\text { PLANEJAMENTO E }\end{array}$ & DESENVOLVIMENTO \\
\hline
\end{tabular}




\begin{tabular}{|c|c|c|c|}
\hline $\begin{array}{c}\text { USO DA TECNOLOGIA DA INFORMAÇÃO NA GESTÃO DA } \\
\text { CADEIA DE SUPRIMENTOS EM SÃO LUÍS, MARANHÃO, E } \\
\text { OPORTUNIDADES PARA O DESENVOLVIMENTO DE } \\
\text { FORNECEDORES LOCAIS }\end{array}$ & 2013 & INTERAÇÕES & $\begin{array}{l}\text { PLANEJAMENTO E } \\
\text { DESENVOLVIMENTO }\end{array}$ \\
\hline $\begin{array}{l}\text { VANTAGENS E OPORTUNISMO NO RELACIONAMENTO } \\
\text { ENTRE ASSOCIADOS E COOPERATIVA DE LATICÍNIOS }\end{array}$ & 2017 & INTERAÇÕES & $\begin{array}{l}\text { PLANEJAMENTO E } \\
\text { DESENVOLVIMENTO }\end{array}$ \\
\hline $\begin{array}{c}\text { SUSTAINABLE FOOD CHAINS: THE ROLE OF COLLECTIVE } \\
\text { ACTION AND GOVERNMENT INCENTIVES }\end{array}$ & 2017 & $\begin{array}{l}\text { ORGANIZAÇÕES E } \\
\text { SOCIEDADE }\end{array}$ & GERENCIAMENTO \\
\hline $\begin{array}{c}\text { EVIDÊNCIAS DA RELAÇÃO ENTRE A EVOLUÇÃO DA } \\
\text { GESTÃO AMBIENTAL E A ADOÇÃO DE PRÁTICAS DE } \\
\text { GREEN SUPLY CHAIN MANAGEMENT NO SETOR } \\
\text { ELETRONICO BRASILEIRO }\end{array}$ & 2014 & $\begin{array}{l}\text { REVISTA DE } \\
\text { ADMINISTRAÇÃO } \\
\text { (SÃO PAULO) }\end{array}$ & GERENCIAMENTO \\
\hline $\begin{array}{l}\text { SHOULD I GO TO COURT? AN ASSESSMENT OF THE } \\
\text { ROLE OF THE JUDICIARY IN DISPUTES BETWEEN CATTLE } \\
\text { RAISERS AND MEATPACKERS IN BRAZIL } \\
\end{array}$ & 2013 & $\begin{array}{l}\text { REVISTA DE } \\
\text { ADMINISTRAÇÃO } \\
\text { (SÃO PAULO) }\end{array}$ & GERENCIAMENTO \\
\hline $\begin{array}{l}\text { ANOTHER DRIVER OF THE BRAZILIAN FUEL ETHANOL } \\
\text { SUPPLY CHAIN: THE CONSUMERS' PREFERENCES }\end{array}$ & 2017 & $\begin{array}{c}\text { REVISTA DE } \\
\text { ADMINISTRAÇÃO } \\
\text { (SÃO PAULO) }\end{array}$ & GERENCIAMENTO \\
\hline $\begin{array}{c}\text { O EFEITO DAS CAPACIDADES LOGÍSTICAS NA } \\
\text { CONSTRUÇÃO DE RESILIÊNCIA DA CADEIA DE } \\
\text { SUPRIMENTOS }\end{array}$ & 2014 & $\begin{array}{l}\text { REVISTA DE } \\
\text { ADMINISTRAÇÃO } \\
\text { (SÃO PAULO) }\end{array}$ & GERENCIAMENTO \\
\hline $\begin{array}{l}\text { BARREIRAS À GESTÃO DA CADEIA DE SUPRIMENTOS } \\
\text { VERDES NA INDÚSTRIA AUTOMOTIVA }\end{array}$ & 2018 & $\begin{array}{l}\text { REVISTA DE } \\
\text { ADMINISTRAÇÃO } \\
\text { DE EMPRESAS }\end{array}$ & GERENCIAMENTO \\
\hline $\begin{array}{l}\text { CADEIA REVERSA DO OLÉO DE COZINHA: } \\
\text { COORDENAÇÃO, ESTRUTURA E ASPECTOS RELACIONAIS }\end{array}$ & 2013 & $\begin{array}{c}\text { REVISTA DE } \\
\text { ADMINISTRAÇÃO } \\
\text { DE EMPRESAS }\end{array}$ & GERENCIAMENTO \\
\hline $\begin{array}{l}\text { IMERSÃO SOCIAL NA CADEIA DE SUPRIMENTOS E SEU } \\
\text { EFEITO PARADOXAL NO DESEMPENHO OPERACIONAL }\end{array}$ & 2014 & $\begin{array}{l}\text { REVISTA DE } \\
\text { ADMINISTRAÇÃO } \\
\text { DE EMPRESAS }\end{array}$ & GERENCIAMENTO \\
\hline $\begin{array}{l}\text { MÉTODOS COLABORATIVOS NA GESTÃO DE CADEIAS } \\
\text { DE SUPRIMENTOS: DESAFIOS DA IMPLEMENTAÇÃOO }\end{array}$ & 2015 & $\begin{array}{c}\text { REVISTA DE } \\
\text { ADMINISTRAÇÃO } \\
\text { DE EMPRESAS }\end{array}$ & GERENCIAMENTO \\
\hline $\begin{array}{l}\text { GREEN SUPPLY CHAIN: PROTAGONISTA OU } \\
\text { COADJUVANTE NO BRASIL? }\end{array}$ & 2014 & $\begin{array}{c}\text { REVISTA DE } \\
\text { ADMINISTRAÇÃO } \\
\text { DE EMPRESAS }\end{array}$ & GERENCIAMENTO \\
\hline $\begin{array}{l}\text { SUPLY CHAIN RESILIENCE ANALYSIS: A BRAZILIAN } \\
\text { AUTOMOTIVE CASE }\end{array}$ & 2014 & $\begin{array}{l}\text { REVISTA DE } \\
\text { ADMINISTRAÇÃO } \\
\text { DE EMPRESAS }\end{array}$ & GERENCIAMENTO \\
\hline $\begin{array}{l}\text { RELACIONAMENTOS COLABORATIVOS E DESEMPENHO } \\
\text { COMPETITIVO DE EMPRESAS BRASILEIRAS }\end{array}$ & 2014 & $\begin{array}{c}\text { REVISTA DE } \\
\text { ADMINISTRAÇÃO } \\
\text { DE EMPRESAS }\end{array}$ & GERENCIAMENTO \\
\hline $\begin{array}{l}\text { NOVAS TECNOLOGIAS E DESEMPENHO OPERACIONAL: } \\
\text { UM ESTUDO INTERNACIONAL COMPARATIVO }\end{array}$ & 2014 & $\begin{array}{l}\text { REVISTA DE } \\
\text { ADMINISTRAÇÃO } \\
\text { DE EMPRESAS }\end{array}$ & GERENCIAMENTO \\
\hline $\begin{array}{l}\text { CORPORATE PROFILE, PERFORMANCE AND GREEN } \\
\text { SUPPLY CHAIN MANAGEMENT: A RESEARCH AGENDA }\end{array}$ & 2017 & RAM & GERENCIAMENTO \\
\hline $\begin{array}{c}\text { DESEMPENHO PRODUTIVO COMO FATOR } \\
\text { MODERADOR DA ESTRATÉGIA } \\
\text { E CAPABILIDADE }\end{array}$ & 2013 & RAM & GERENCIAMENTO \\
\hline
\end{tabular}




\begin{tabular}{|c|c|c|c|}
\hline $\begin{array}{l}\text { GREEN MARKETING AS A MEDIATOR } \\
\text { BETWEEN SUPPLY CHAIN MANAGEMENT } \\
\text { AND ORGANIZATIONAL PERFORMANCE }\end{array}$ & 2016 & RAM & GERENCIAMENTO \\
\hline $\begin{array}{c}\text { ROLE OF TECHNOLOGY IN THE PERFORMANCE OF THE } \\
\text { BRAZILIAN CHEMICAL INDUSTRY }\end{array}$ & 2018 & RAM & GERENCIAMENTO \\
\hline $\begin{array}{c}\text { APLICAÇÃO DA METODOLOGIA MULTICRITÉRIO DE } \\
\text { APOIO À DECISÃO NO RELACIONAMENTO } \\
\text { INTERORGANIZACIONAL NA CADEIA DA AVICULTURA } \\
\text { DE CORTE }\end{array}$ & 2016 & $\begin{array}{l}\text { ECONOMIA E } \\
\text { SOCIOLOGIA } \\
\text { RURAL }\end{array}$ & $\begin{array}{c}\text { ECONOMIA E } \\
\text { POLÍTICA AGRÍCOLA }\end{array}$ \\
\hline $\begin{array}{c}\text { ALINHAMENTO ESTRATÉGICO NAS CADEIAS DE } \\
\text { SUPRIMENTO DA INDÚSTRIA AUTOMOBILÍSTICA } \\
\text { BRASILEIRA }\end{array}$ & 2012 & $\begin{array}{c}\text { REVISTA } \\
\text { ELETRÔNICA DE } \\
\text { ADMINISTRAÇÃO }\end{array}$ & GERENCIAMENTO \\
\hline $\begin{array}{l}\text { ANÁLISE DA RESTRIÇÃO DA OFERTA E INSUMOS } \\
\text { BÁSICOS EM PERIÓDOS ECONÔMICOS FAVORÁVEIS DA } \\
\text { PERSPETICA DE UM FABRICANTE DE AVIÃO }\end{array}$ & 2014 & $\begin{array}{c}\text { REVISTA } \\
\text { ELETRÔNICA DE } \\
\text { ADMINISTRAÇÃO }\end{array}$ & GERENCIAMENTO \\
\hline $\begin{array}{l}\text { ANÁLISE DA UTILIZAÇÃO DE TECNOLOGIAS DA } \\
\text { INFORMAÇÃO MÓVEIS E SEM FIO (TIMS) NA CADEIA } \\
\text { BOVINA: UM ESTUDO DE CASO NO ESTADO DE GOIÁS }\end{array}$ & 2014 & $\begin{array}{c}\text { REVISTA } \\
\text { ELETRÔNICA DE } \\
\text { ADMINISTRAÇÃO }\end{array}$ & GERENCIAMENTO \\
\hline $\begin{array}{l}\text { E-PROCUREMENT: CONCEITOS, MODELOS E FATORES } \\
\text { CRÍTICOS DE SUCESSO }\end{array}$ & 2016 & $\begin{array}{c}\text { REVISTA } \\
\text { ELETRÔNICA DE } \\
\text { ADMINISTRAÇÃO }\end{array}$ & GERENCIAMENTO \\
\hline $\begin{array}{l}\text { O EFEITO DO TIPO DE PRODUTO E DAS CAPABILIDADES } \\
\text { LOGÍSTICAS SOBRE O CÍCLO DO PEDIDO }\end{array}$ & 2015 & $\begin{array}{c}\text { REVISTA } \\
\text { ELETRÔNICA DE } \\
\text { ADMINISTRAÇÃO }\end{array}$ & GERENCIAMENTO \\
\hline $\begin{array}{l}\text { MUDANÇAS NAS PRÁTICAS ORGANIZACIONAIS COM A } \\
\text { IMPLEMENTAÇÃO DO PROGRAMA DE } \\
\text { GERENCIAMENTO DA CADEIA DE SUPRIMENTOS (GCS) } \\
\text { EM UMA MULTINACIONAL DO SETOR SIDERÚRGICO }\end{array}$ & 2012 & $\begin{array}{c}\text { REVISTA DE } \\
\text { GESTÃO DA } \\
\text { TECNOLOGIA E } \\
\text { SISTEMAS DE } \\
\text { INFORMAÇÃO }\end{array}$ & GERENCIAMENTO \\
\hline $\begin{array}{l}\text { A CASE-BASED ROADMAP FOR LATERAL } \\
\text { TRANSSHIPMENT IN SUPPLY CHAIN INVENTORY } \\
\text { MANAGEMENT }\end{array}$ & 2016 & $\begin{array}{c}\text { REVISTA DE } \\
\text { GESTÃO DA } \\
\text { TECNOLOGIA E } \\
\text { SISTEMAS DE } \\
\text { INFORMAÇÃO }\end{array}$ & GERENCIAMENTO \\
\hline $\begin{array}{l}\text { MANAGEMENT MODEL FOR THE DEVELOPMENT OF } \\
\text { SOFTWARE APPLIED TO BUSINESS SUSTAINABILITY IN } \\
\text { THE CONTEXT OF GLOBAL CLIMATE CHANGES }\end{array}$ & 2012 & $\begin{array}{c}\text { REVISTA DE } \\
\text { GESTÃO DA } \\
\text { TECNOLOGIA E } \\
\text { SISTEMAS DE } \\
\text { INFORMAÇÃO }\end{array}$ & GERENCIAMENTO \\
\hline $\begin{array}{c}\text { AS DIMENSÕES ESPACIAIS DA CADEIA PRODUTIVA } \\
\text { DO LEITE EM MINAS GERAIS }\end{array}$ & 2018 & NOVA ECONOMIA & ECONOMIA \\
\hline $\begin{array}{c}\text { SECTORAL CAPABILITIES AND PRODUCTIVE } \\
\text { STRUCTURE: } \\
\text { AN INPUT-OUTPUT ANALYSIS OF THE KEY SECTORS } \\
\text { OF THE BRAZILIAN ECONOMY }\end{array}$ & 2016 & $\begin{array}{l}\text { BRAZILIAN } \\
\text { JOURNAL OF } \\
\text { POLITICAL } \\
\text { ECONOMY }\end{array}$ & ECONOMIA \\
\hline $\begin{array}{c}\text { A PRÁTICA DA GESTÃO DE CUSTOS } \\
\text { INTERORGANIZACIONAIS EM UMA MONTADORA DE } \\
\text { VEÍCULOS NA REGIÃO DO GRANDE ABC }\end{array}$ & 2013 & $\begin{array}{l}\text { REVISTA } \\
\text { BRASILEIRA DE } \\
\text { GESTÃO E } \\
\text { NEGÓCIOS }\end{array}$ & GERENCIAMENTO \\
\hline $\begin{array}{c}\text { ALIMENTAÇÃO, SISTEMA AGROALIMENTAR E } \\
\text { OS CONSUMIDORES: NOVAS CONEXÕES PARA O } \\
\text { DESENVOLVIMENTO RURAL }\end{array}$ & 2015 & $\begin{array}{l}\text { CUADERNOS DE } \\
\text { DESARROLLO } \\
\text { RURAL }\end{array}$ & AGRICULTURA \\
\hline $\begin{array}{l}\text { A CADEIA PRODUTIVA DO TABACO COMO } \\
\text { CAMPO DE DISPUTAS }\end{array}$ & 2017 & MUNDO AGRARIO & AGRICULTURA \\
\hline
\end{tabular}




\begin{tabular}{|c|c|c|c|}
\hline $\begin{array}{l}\text { COLLABORATIVE PLATFORMS FOR SUPPLY CHAIN } \\
\text { INTEGRATION: TRAJECTORY, ASSIMILATION } \\
\text { OF PLATFORMS AND RESULTS }\end{array}$ & 2015 & $\begin{array}{l}\text { JOURNAL OF } \\
\text { TECHNOLOGY } \\
\text { MANAGEMENT \& } \\
\text { INNOVATION }\end{array}$ & ENGENHARIA \\
\hline $\begin{array}{c}\text { INNOVATION AND SUSTAINABILITY IN THE SUPPLY } \\
\text { CHAIN OF A COSMETICS COMPANY: } \\
\text { A CASE STUDY }\end{array}$ & 2012 & $\begin{array}{l}\text { JOURNAL OF } \\
\text { TECHNOLOGY } \\
\text { MANAGEMENT \& } \\
\text { INNOVATION } \\
\end{array}$ & ENGENHARIA \\
\hline $\begin{array}{c}\text { MAPPING SUSTAINABLE STRUCTURAL DIMENSIONS } \\
\text { FOR MANAGING THE BRAZILIAN } \\
\text { BIODIESEL SUPPLY CHAIN }\end{array}$ & 2014 & $\begin{array}{l}\text { JOURNAL OF } \\
\text { TECHNOLOGY } \\
\text { MANAGEMENT \& } \\
\text { INNOVATION } \\
\end{array}$ & ENGENHARIA \\
\hline $\begin{array}{l}\text { PROCEDURAL PRIORITIES OF THE PORK LOIN SUPPLY } \\
\text { CHAIN }\end{array}$ & 2014 & $\begin{array}{l}\text { JOURNAL OF } \\
\text { TECHNOLOGY } \\
\text { MANAGEMENT \& } \\
\text { INNOVATION } \\
\end{array}$ & ENGENHARIA \\
\hline $\begin{array}{c}\text { SUPPLY NETWORKS AND VALUE CREATION IN HIGH } \\
\text { INNOVATION AND STRONG NETWORK } \\
\text { EXTERNALITIES INDUSTRY }\end{array}$ & 2013 & $\begin{array}{l}\text { JOURNAL OF } \\
\text { TECHNOLOGY } \\
\text { MANAGEMENT \& } \\
\text { INNOVATION } \\
\end{array}$ & ENGENHARIA \\
\hline $\begin{array}{l}\text { TECHNOLOGICAL CAPABILITIES OF BRAZILIAN } \\
\text { SHIPBUILDING SUPPLIERS }\end{array}$ & 2012 & $\begin{array}{l}\text { JOURNAL OF } \\
\text { TECHNOLOGY } \\
\text { MANAGEMENT \& } \\
\text { INNOVATION } \\
\end{array}$ & ENGENHARIA \\
\hline $\begin{array}{l}\text { SUSTAINABILITY INITIATIVES DRIVING SUPPLY CHAIN: } \\
\text { CLIMATE GOVERNANCE ON BEEF PRODUCTION SYSTEM }\end{array}$ & 2015 & $\begin{array}{l}\text { JOURNAL OF } \\
\text { TECHNOLOGY } \\
\text { MANAGEMENT \& } \\
\text { INNOVATION } \\
\end{array}$ & ENGENHARIA \\
\hline $\begin{array}{l}\text { A PLURALIDADE NAS TRANSAÇÕES DE CANA-DE- } \\
\text { AÇÚCAR NO OESTE PAULISTA }\end{array}$ & 2013 & $\begin{array}{c}\text { REVISTA DE } \\
\text { ADMINISTRAÇÃO } \\
\text { CONTEMPORÂNEA } \\
\end{array}$ & GERENCIAMENTO \\
\hline $\begin{array}{l}\text { EVALUATING SUPPLY CHAIN MANAGEMENT: A } \\
\text { METHODOLOGY BASED ON A THEORETICAL MODEL }\end{array}$ & 2015 & $\begin{array}{c}\text { REVISTA DE } \\
\text { ADMINISTRAÇÃO } \\
\text { CONTEMPORÂNEA }\end{array}$ & GERENCIAMENTO \\
\hline $\begin{array}{l}\text { INFORMATION TECHNOLOGY INVESTMENTS AND } \\
\text { SUPPLY CHAIN GOVERNANCE }\end{array}$ & 2014 & $\begin{array}{c}\text { REVISTA DE } \\
\text { ADMINISTRAÇÃO } \\
\text { CONTEMPORÂNEA }\end{array}$ & GERENCIAMENTO \\
\hline $\begin{array}{l}\text { OLIGOPSÔNIO DOS FRIGORÍFICOS: UMA ANÁLISE } \\
\text { EMPÍRICA DE PODER DE MERCADO }\end{array}$ & 2014 & $\begin{array}{c}\text { REVISTA DE } \\
\text { ADMINISTRAÇÃO } \\
\text { CONTEMPORÂNEA }\end{array}$ & GERENCIAMENTO \\
\hline $\begin{array}{c}\text { SÍNTESE DOS PRINCIPAIS CRITÉRIOS, MÉTODOS E } \\
\text { SUBPROBLEMAS DA SELEÇÃO DE FORNECEDORES } \\
\text { MULTICRITÉRIO }\end{array}$ & 2015 & $\begin{array}{c}\text { REVISTA DE } \\
\text { ADMINISTRAÇÃO } \\
\text { CONTEMPORÂNEA }\end{array}$ & GERENCIAMENTO \\
\hline $\begin{array}{l}\text { VALOR E VANTAGEM COMPETITIVA: BUSCANDO } \\
\text { DEFINIÇÕES, RELAÇÕES E REPERCUSSÕES }\end{array}$ & 2012 & $\begin{array}{c}\text { REVISTA DE } \\
\text { ADMINISTRAÇÃO } \\
\text { CONTEMPORÂNEA }\end{array}$ & GERENCIAMENTO \\
\hline $\begin{array}{c}\text { A REDE BRASILEIRA DE PRODUÇÃO PÚBLICA DE } \\
\text { MEDICAMENTOS NA } \\
\text { PERSPECTIVA DA GESTÃO DE CADEIAS DE } \\
\text { SUPRIMENTOS: O PAPEL DAS } \\
\text { TIC }\end{array}$ & 2014 & $\begin{array}{l}\text { REVISTA DE } \\
\text { ADMINISTRAÇÃO } \\
\text { PÚBLICA }\end{array}$ & $\begin{array}{l}\text { ADMINISTRAÇÃO } \\
\text { PÚBLICA }\end{array}$ \\
\hline $\begin{array}{c}\text { ELEMENTOS INSTITUCIONAIS E DESEMPENHO DA } \\
\text { LOGÍSTICA DE UMA REDE } \\
\text { PÚBLICA DE ASSISTÊNCIA FARMACÊUTICA }\end{array}$ & 2016 & $\begin{array}{c}\text { REVISTA DE } \\
\text { ADMINISTRAÇÃO } \\
\text { PÚBLICA } \\
\end{array}$ & $\begin{array}{l}\text { ADMINISTRAÇÃO } \\
\text { PÚBLICA }\end{array}$ \\
\hline
\end{tabular}


